\title{
Accuracy of Fitbit Activity Trackers During Walking in a Controlled Setting
}

JOSÉ L. GÁMEZ, JESÚS P. GONZÁLEZ, PERLA LEYVA, IVÁN A. FIGUEROA, NAOMI LUCIO, VANESSA E. SALAZAR, CINDY SALAZAR, MIRIAM GARCIA and MERRILL D. FUNK.

Exercise Science Lab; Department of Health and Human Performance; University of Texas Rio Grande Valley; Brownsville TX University of Texas School of Public Health-Health Science Center at Houston Brownsville Regional Campus

Category: Masters

Advisor / Mentor: Funk, Merrill (Merrill.funk@utrgv.edu)

ABSTRACT

Activity trackers are widely used to measure daily physical activity. Many devices have been shown to measure steps more accurately at higher intensities, however, it is also important to determine the accuracy of these new devices at measuring steps while walking at a pace similar to that used during most daily activities. PURPOSE: To assess the accuracy of 6 popular activity trackers at measuring steps while walking on a treadmill. METHODS: Twenty-six college students (Mean \pm SD; $22.1 \pm 3.7 \mathrm{yrs} ; 25.1 \pm 4.0 \mathrm{~kg} / \mathrm{m}^{2} ; 13$ male) walked 500 steps at $3 \mathrm{mph}$ on a treadmill while wearing 6 different activity trackers (Pedometer, Fitbit Blaze, Charge HR, Alta, Flex, Zip, One). The Charge HR was placed two fingers above the right wrist while the Flex was next to the wrist bone. The Blaze was placed two fingers above the left wrist while the Alta was next to the wrist bone. The Fitbit Zip and the One were aligned with the hipbone on the left and right waistband respectively. Steps were counted by a trained researcher using a hand tally counter. Missing values were replaced with the mean value for that device. Step counts were correlated between Fitbit devices and the pedometer and tally counter using Pearson correlations. Significance was set at $p<0.05$. Mean bias scores were calculated between the step counts for each device and the tally counter. Mean Absolute Percent Error (MAPE) values were also calculated for each device relative to the tally counter. RESULTS: Fitbit Zip and One were significantly correlated with the tally counter $(r=0.50, p<0.05 ; r=0.68, p<0.01$, respectively) while the other devices were not significantly correlated. Mean bias and MAPE values were as follows: Device (Mean Bias/MAPE) Pedometer $(-0.2 \pm 39.2 / 3.8 \pm 6.8)$, Blaze $(34.5 \pm 67.1 / 9.9 \pm 11.3)$, Charge HR (-

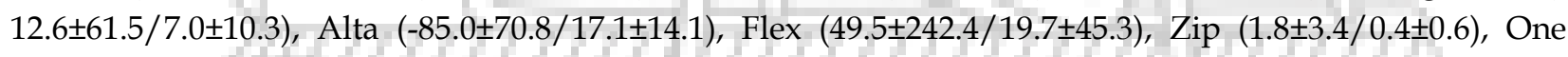
$(0.2 \pm 2.1 / 0.3 \pm 0.3)$. Fitbit Zip and One were within one half percent of actual steps while wrist-worn Fitbits ranged from 7.0-19.7\% from actual step counts. CONCLUSION: Consistent with previous research, activity trackers worn at the waist provide the most accurate step counts compared to wrist-worn models. Differences found in wrist-worn models may result in significant over- or underestimation of activity levels when worn for long periods of time. 